\title{
A Comparison of Frequency Pullability in Oscillators Using a Single AT-Cut Quartz Crystal and Those Using Two Single AT- Cut Crystals Connected in Parallel with a Series Load Capacitance or Series Load Inductance
}

\author{
Vojko Matko \\ University of Maribor, Faculty of Electrical Engineering and Computer Science, Smetanova 17, 2000 \\ Maribor, Slovenia \\ E-mail: vojko.matko@uni-mb.si
}

Received: 6 April 2006 / Accepted: 5 July 2006 / Published: 29 July 2006

\begin{abstract}
This paper presents a comparison of frequency pullability in oscillators using a single AT-cut crystal and those using two single AT-cut crystals connected in parallel operated with a series load capacitance or series load inductance at fundamental frequencies of 4, 10 and $19 \mathrm{MHz}$. Pullability describes how the operating frequency may be changed by varying the load capacitance. The paper also gives impedance circuits for both single- and dual-crystal units. The experiment results show that the new approach using two single quartz crystals connected in parallel increases the frequency pulling range by $30-200 \%$ depending on the type of oscillator. Also given is the crystal frequency stability at these three frequencies.
\end{abstract}

Keywords: quartz, AT cut, pulling range, two single-crystal unit oscillator.

\section{Introduction}

There are many different types of oscillators using crystals as the key components of their circuit. Quartz, in particular, is uniquely suited for the manufacture of frequency selection or frequency control devices. In oscillators with load capacitance in series or parallel with the crystal unit, the oscillation frequency depends on the capacitive load that is applied. The frequency will increase if the capacitive load is decreased and decrease if the load is increased. The amount of frequency change (in ppm) as a function of load capacitance is referred to as the pullability. It indicates how far from the nominal frequency (intended oscillating frequency) the resonant frequency can be forced by applying 
the load. Typically, it is used to tune the operating frequency to a desired value. In special cases, it can also be used for the measurement purposes allowing the measurement of various quantities based on capacitive and inductive influence on the quartz crystal oscillation frequency.

This research focuses on the influence of the series load capacitance and load inductance on the pullability using AT-cut quartz crystals (cut angle: +4 ') operating over the temperature range of $-10^{\circ} \mathrm{C}$ to $+40^{\circ} \mathrm{C}$. Crystals fabricated in this manner exhibit excellent frequency vs temperature stability. They have fundamental resonant frequencies between 1 and $40 \mathrm{MHz}$. Fundamental mode crystals (especially those housed in the familiar HC-49/U holder) exhibit a higher sensitivity to frequency pulling than overtone mode crystals. Moreover, low frequency crystals provide higher quality factor $Q$ and achieve greater frequency stability than higher frequency fundamental crystals. The principal advantage of ATcut over other cuts is the low frequency sensitivity to change in temperature.

The operation of a quartz crystal is frequently explained using the familiar "Equivalent Circuit", illustrated in Fig.1 representing an electrical depiction of the quartz crystal unit [1-3].

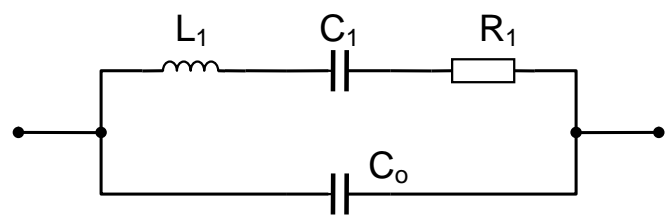

Figure 1. The quartz crystal equivalent circuit.

In Fig. 1, the capacitance labeled " $\mathrm{C}_{0}$ " is a real capacitance, comprising the capacitance between the electrodes and the stray capacitance associated with the mounting structure. It is also known as the "shunt" or "static" capacitance, and represents the crystal in a non-operational, or static, state. The other components represent the crystal in an operational or motional state: " $L_{1}$ ", " $C_{1}$ ", and " $R_{1}$ ", identify the "motional inductance", the "motional capacitance", and the "motional resistance", respectively. The motional inductance $\mathrm{L}_{1}$ represents the vibrating mass of the quartz plate, while the motional capacitance $C_{1}$ represents the elasticity or stiffness of the plate. The motional resistance $R_{1}$, often simply called the "resistance", represents the bulk losses occurring within the vibrating plate.

Conventional crystal units (such as those packaged in the HC-49/U holder) typically use a circular quartz resonator plate equipped with circular electrodes. The electrodes are applied to the surface of the quartz plate using metal deposition under vacuum. Proper placement is ensured through the use of masks that cover all of the plate except the area to be electroded. The masks are usually made of three parts: a center part with nests for the plate, and upper and lower parts that provide the apertures for the electrode. When making such masks, it is easy to change the aperture that determines the electrode's size; thus a wide variety of electrode sizes can be applied to a resonator plate of specific diameter. As noted above, the size of the electroded area determines the crystal's motional parameters, and it is thus possible to specify those parameters to fit the part to a specific application.

There are two resonance frequencies, the series resonance frequency $f_{S}$ and the parallel resonance frequency $f_{P}$.

$$
f_{s}=\frac{1}{2 \pi \cdot \sqrt{L_{1} \cdot C_{1}}}
$$




$$
f_{p}=\frac{1}{2 \pi \cdot \sqrt{L_{1} \frac{C_{1} \cdot C_{0}}{C_{1}+C_{0}}}}=f_{s} \sqrt{1+\frac{C_{1}}{C_{0}}}
$$

The series and parallel resonance frequencies are related by the formula

$$
\frac{f_{p}-f_{s}}{f_{s}} \approx \frac{1}{2} \cdot \frac{C_{1}}{C_{0}}
$$

The quality factor $Q$ of the quartz crystal unit as a measure of the unit's relative quality, or efficiency of oscillation, is specified as

$$
Q=\frac{2 \pi \cdot f_{s} \cdot L_{1}}{R_{1}}=\frac{1}{2 \pi \cdot f_{s} \cdot R_{1} \cdot C_{1}}
$$

The complex impedance equation for the crystal equivalent circuit (Fig.1) is [1]

$$
\bar{Z}=\frac{\left(R_{1}+j \omega L_{1}+\frac{1}{j \omega C_{1}}\right) \cdot \frac{1}{j \omega C_{0}}}{R_{1}+j \omega L_{1}+\frac{1}{j \omega C_{1}}+\frac{1}{j \omega C_{0}}}
$$

\section{Quartz Crystal Unit with Series Load Capacitance $C_{L}$ and Series Load Inductance $L_{L}$}

Fundamental mode quartz crystals are normally operated with a load capacitance, which allows the circuit capacitance variations to be compensated. For example, for an application requiring a crystal with high pullability, it is simple to apply electrodes that result in such a resonator. Conversely, if pullability is to be avoided, electrodes that avoid this condition can be easily designed. If the electrode required by the application is as large as or even larger than the resonator plate, one can often use a somewhat larger plate in the specified holder [4].

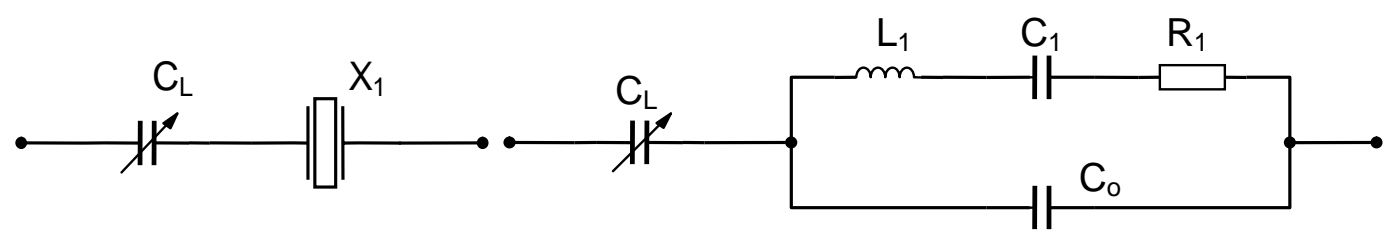

Figure 2. Quartz crystal unit operated with a load capacitance.

As the capacitive load in series with the crystal is varied, the crystal frequency is pulled (Fig. 2). This change of the frequency with load capacitance $C_{L}$ is expressed by 


$$
f_{L}=f_{S} \cdot \sqrt{1+\frac{C_{1}}{C_{0}+C_{L}}} \approx f_{S}\left(1+\frac{C_{1}}{2\left(C_{0}+C_{L}\right)}\right)
$$

where $f_{L}$ is frequency at given load capacitance $\mathrm{C}_{\mathrm{L}}$

and

$\frac{f_{L}-f_{S}}{f_{S}}=\frac{C_{1}}{2\left(C_{0}+C_{L}\right)}$

The pulling range $D_{C L 1, C L 2}$ of the element is defined as the change in frequency produced by changing the load capacitance from one value to another (Fig. 2).

$$
D_{C L 1, C L 2}=\frac{f_{C L 1}-f_{C L 2}}{f_{S}}=\frac{C_{1}\left(C_{L 2}-C_{L 1}\right)}{2 \cdot\left(C_{0}+C_{L 1}\right)\left(C_{0}+C_{L 2}\right)}
$$

We can define pulling sensitivity $S$ as the frequency change in parts per million (ppm) per pF change in the load capacitance

$$
S=\frac{1}{f_{r}} \cdot \frac{d f_{C L}}{d C_{L}} \approx \frac{C_{1}}{2\left(C_{0}+C_{L}\right)^{2}}
$$

where $f_{r}$ is resonance frequency with phase 0 .

The pulling sensitivity if quartz crystal unit is operated with a load inductance (Fig. 3) is defined as

$$
S=\frac{1}{f_{r}} \cdot \frac{d f_{L}}{d L_{L}}
$$

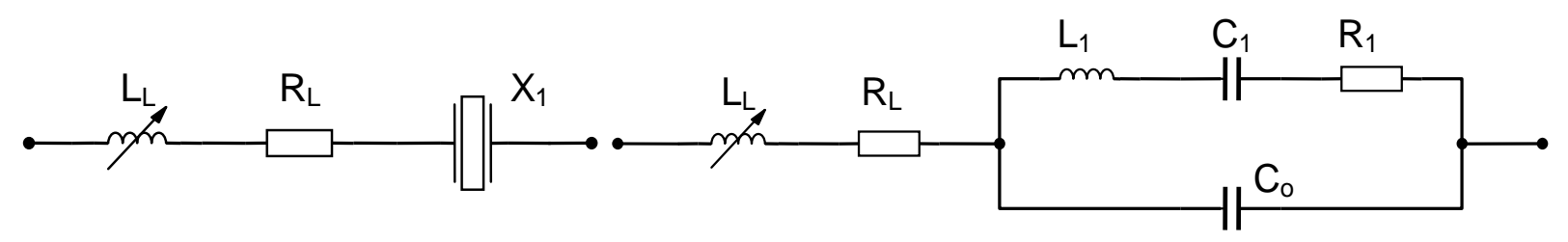

Figure 3. Quartz crystal unit operated with a load inductance.

In both cases, the increase in the capacitance ratio $\left(C_{0} / C_{1}\right)$ decreases the frequency change $\left(f_{L}-f_{S}\right) / f_{S}$, thus reducing the pulling range of a crystal unit.

The maximum attainable stability of a crystal unit is dependent on the $Q$ value. The smaller the distance between $\mathrm{f}_{\mathrm{s}}$ and $\mathrm{f}_{\mathrm{p}}$, the higher the $Q$ value, and the steeper the slope of the reactance. Changes in the reactance of external circuit components have less effect, less "pullability", on a crystal with high $Q$ factor. Therefore such a part is more stable. Smaller crystals have about half the pullability of 
the HC-49/U. The pullability of overtone crystal is reduced by $1 / \mathrm{n}^{2}$, where $\mathrm{n}$ is the overtone mode (i.e. $1,3,5$, etc.) $[1,4,5]$.

\section{Experimental Circuit with Two Single Crystals Connected in Parallel}

Low cost Transistor-Transistor Logic (TTL) oscillator is a commonly used circuit employing invertors or gates with AT-cut crystals. Due to the low cost of components this is a popular circuit.

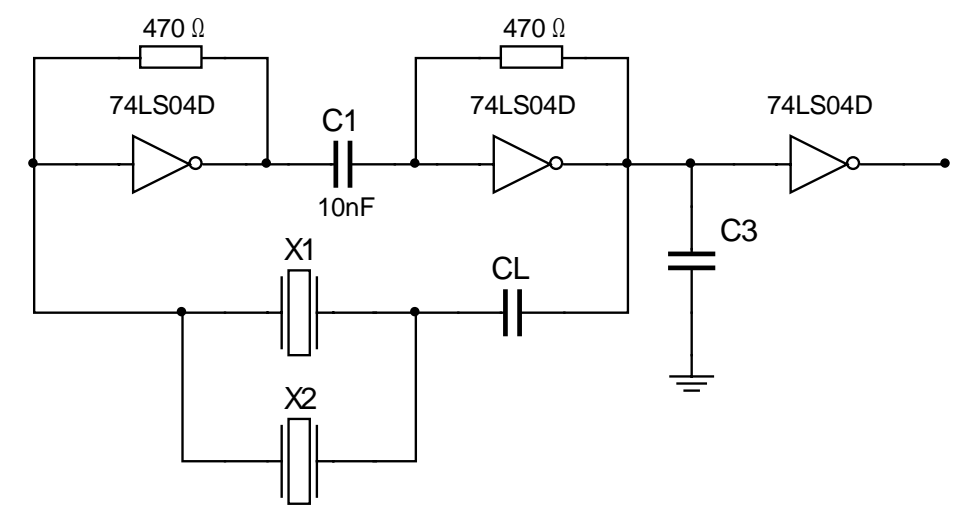

Figure 4. Low-cost TTL oscillator.

Capacitor $\mathrm{C}_{\mathrm{L}}$ (Fig. 4) (which can be a load capacitor) is intended to cancel the effective series resistance of the invertors. Unfortunately, the lagging phase shift problem is aggravated by the presence of $\mathrm{C}_{3}$, which is necessary to prevent fast wave fronts from exciting the crystal third-overtone mode; this can be a nuisance below $8 \mathrm{MHz}$. Both crystals are the same frequency. The swing obtainable by adding the second crystal can be considerable - measurements show an increase in pulling range [5-6].

\section{Experimental Results}

Table 1 lists the parameters of the crystals used in the experiment and Fig. 5 shows their impedance circles. The values in the quartz crystal equivalent circuit were measured by the HP 4194A impedance/gain-phase analyzer.

Table 1. Quartz data.

\begin{tabular}{|c|c|r|c|c|c|}
\hline $\mathrm{f}$ & $\mathrm{C}_{1}$ & \multicolumn{1}{c|}{$\mathrm{L}_{1}$} & $\mathrm{R}_{1}$ & $\mathrm{C}_{0}$ & $\mathrm{Q}$ \\
\hline $4 \mathrm{MHz}$ & $10 \mathrm{fF}$ & $158.31 \mathrm{mH}$ & $50 \Omega$ & $4.5 \mathrm{pF}$ & $7.161 \cdot 10^{6}$ \\
\hline $10 \mathrm{MHz}$ & $20 \mathrm{fF}$ & $12.66 \mathrm{mH}$ & $12 \Omega$ & $4.5 \mathrm{pF}$ & $1.432 \cdot 10^{6}$ \\
\hline $19 \mathrm{MHz}$ & $21 \mathrm{fF}$ & $3.34 \mathrm{mH}$ & $6.1 \Omega$ & $4.5 \mathrm{pF}$ & $0.718 \cdot 10^{6}$ \\
\hline
\end{tabular}

If we define the frequency ratio $\Omega=\omega / \omega_{0}$, which depends on $\omega_{0}=1 / \sqrt{L_{1} \cdot C_{1}}$, and taking into account $\omega_{0} L_{1}=1 / \omega_{0} C_{1}$, the impedance equation for a single crystal unit is [1] 
$\bar{Z}_{q}(\Omega)=R \frac{1+j \frac{\omega_{0} \cdot L_{1}}{R_{1}}\left(\Omega-\frac{1}{\Omega}\right)}{1+\frac{C_{0}}{C_{1}}\left(1-\Omega^{2}\right)+j \frac{C_{0}}{C_{1}} \cdot \frac{R_{1}}{\omega_{0} \cdot L_{1}} \cdot \Omega}$.

The impedance equation for two single quartz crystals connected in parallel can be written as a complex substitutional equation for both crystals

$$
\bar{Z}_{q q}(\Omega)=\frac{\bar{Z}_{q}(\Omega) \cdot \bar{Z}_{q}(\Omega)}{\bar{Z}_{q}(\Omega)+\bar{Z}_{q}(\Omega)} .
$$

$\Omega:=0.999,0.9990010 \ldots 1.006$

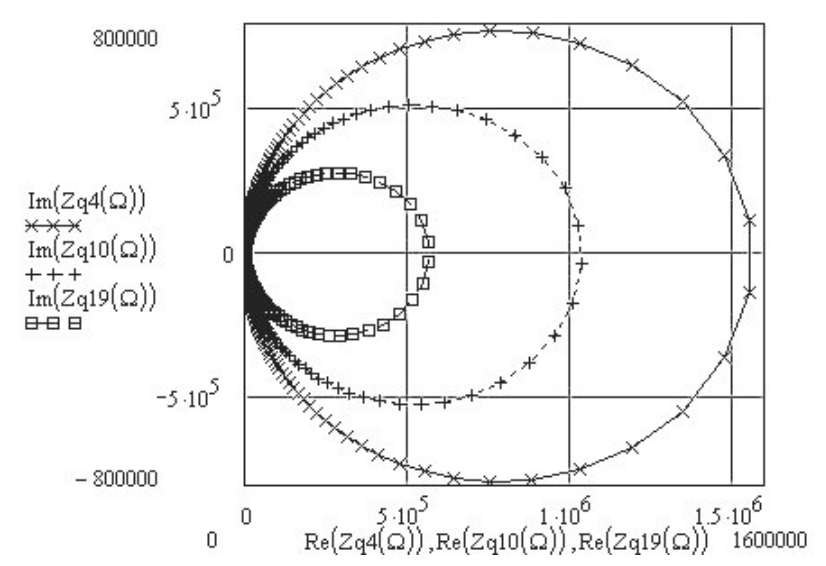

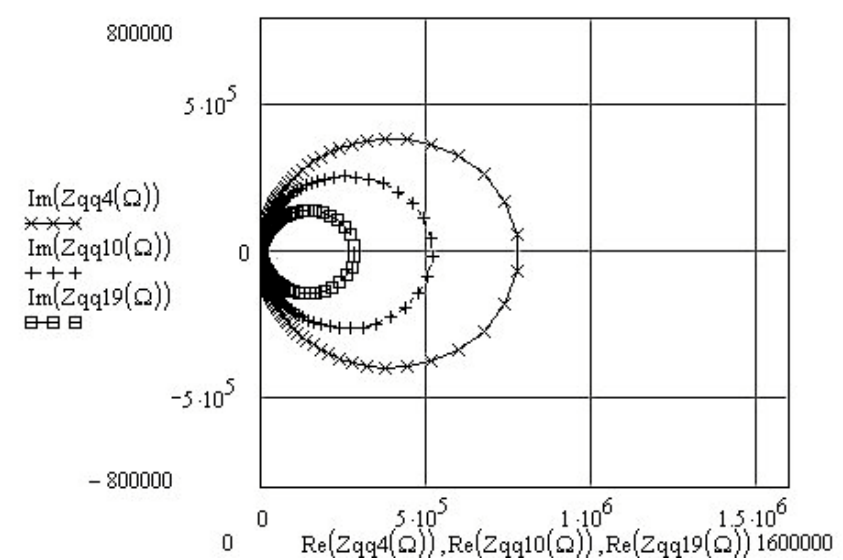

Figure 5. Impedance circles for the oscillation frequencies of 4, 10 and $19 \mathrm{MHz}$ for a single- and dualcrystal unit.
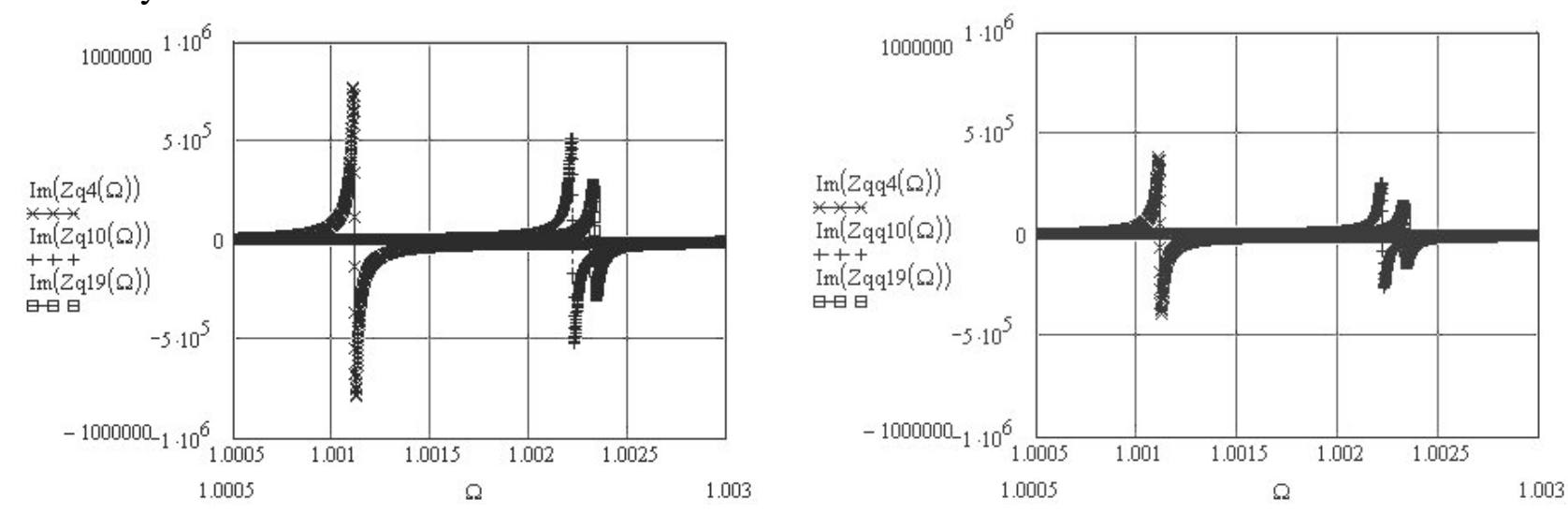

Figure 6. Phase diagrams for a single- and dual-crystal unit operating at the oscillation frequencies of 4, 10 and $19 \mathrm{MHz}$.

The equation in the case of capacitively pulled single-crystal unit can be written as [1]

$\frac{1}{j \omega \cdot C_{L}}=\frac{\sqrt{L_{1} \cdot C_{1}}}{j \Omega \cdot C_{L}}$ 
and

$$
\bar{Z}_{C_{L} q}(\Omega)=R \frac{1+j \frac{\omega_{0} \cdot L_{1}}{R_{1}}\left(\Omega-\frac{1}{\Omega}\right)}{1+\frac{C_{0}}{C_{1}}\left(1-\Omega^{2}\right)+j \frac{C_{0}}{C_{1}} \cdot \frac{R_{1}}{\omega_{0} \cdot L_{1}} \cdot \Omega}+\frac{\sqrt{L_{1} \cdot C_{1}}}{j \Omega \cdot C_{L}} .
$$

In the case of capacitively pulled dual-crystal unit the equation is

$$
\bar{Z}_{C_{L} q q}(\Omega)=\frac{\bar{Z}_{q}(\Omega) \cdot \bar{Z}_{q}(\Omega)}{\bar{Z}_{q}(\Omega)+\bar{Z}_{q}(\Omega)}+\frac{\sqrt{L_{1} \cdot C_{1}}}{j \Omega \cdot C_{L}} .
$$

To compare the amount of pullability exhibited by a given crystal unit, the oscillator frequency was measured by the Heterodyne method, where $d f=f_{1}-f_{2}$ [7-8]. This is the reason why the results are shown in the frequency range $0-20 \mathrm{kHz}$ (Fig. 7-9). For the experimental measurement of pullability exhibited by a crystal unit operated in series with load capacitance the ceramic capacitors with the temperature coefficient 0 were used. Capacitor values were measured using the HP 4194A impedance/gain-phase analyzer.
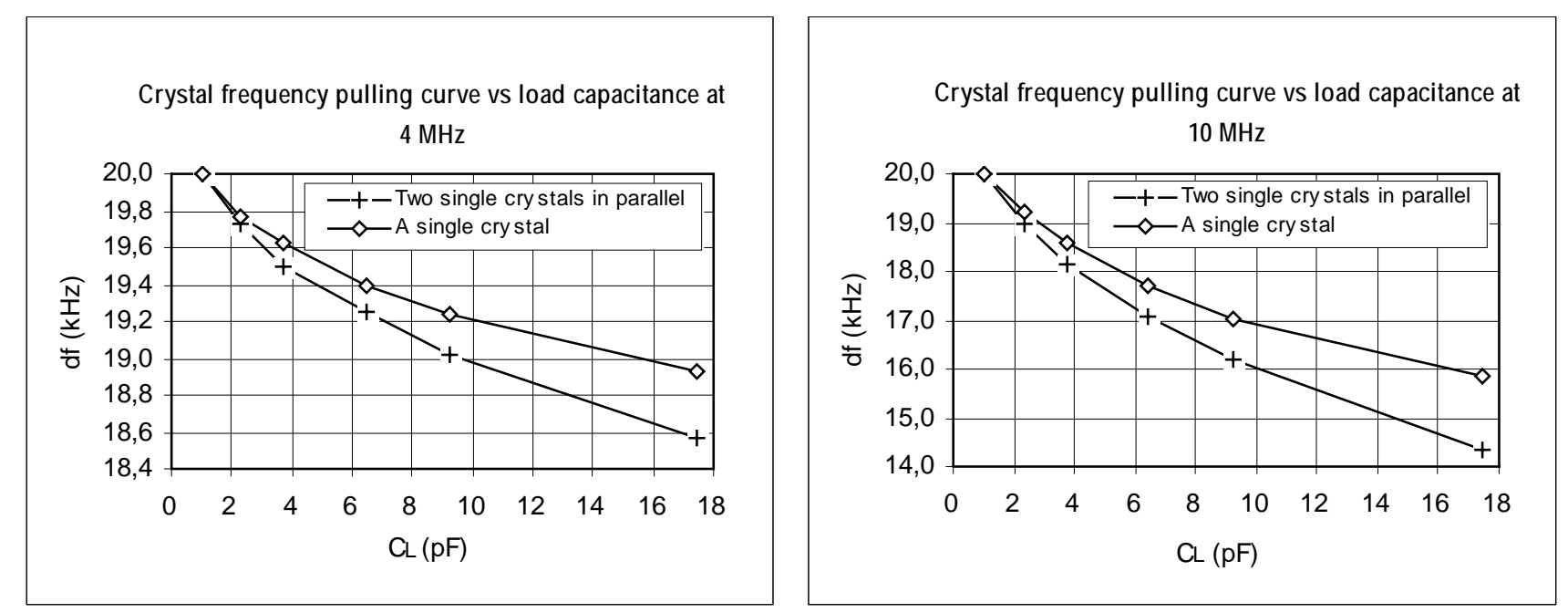

Figure 7. A comparison of pullability exhibited by a single- and dual-crystal unit operated in series with load capacitance at the frequencies of 4 and $10 \mathrm{MHz}$.

Using equation (16), the impedance equation for an inductively pulled single-crystal unit can be written as equation (17).

$$
j \omega L_{L}+R_{L}=j \frac{\Omega \cdot L_{L}}{\sqrt{L_{1} \cdot C_{1}}}+R_{L}
$$

where $R_{L}$ is the real part ( $\sim 80 \mathrm{~m} \Omega$ ) of the impedance of the coil $(1 \mu \mathrm{H})$ 


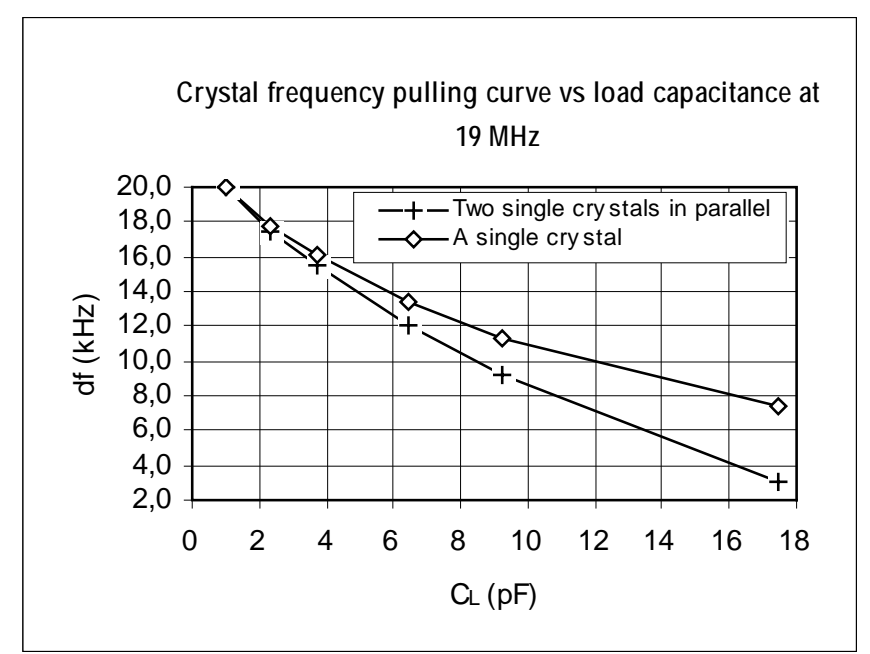

Figure 8. A comparison of pullability exhibited by a single- and dual-crystal unit operated in series with load capacitance at the frequency of $19 \mathrm{MHz}$.

$\bar{Z}_{L_{L} q}(\Omega)=R \frac{1+j \frac{\omega_{0} L_{1}}{R_{1}}\left(\Omega-\frac{1}{\Omega}\right)}{1+\frac{C_{0}}{C_{1}}\left(1-\Omega^{2}\right)+j \frac{C_{0}}{C_{1}} \cdot \frac{R_{1}}{\omega_{0} L_{1}} \cdot \Omega}+j \frac{\Omega \cdot L_{L}}{\sqrt{L_{1} \cdot C_{1}}}+R_{L}$.

The impedance equation for two single crystals is

$\bar{Z}_{L_{L} q q}(\Omega)=\frac{\bar{Z}_{q}(\Omega) \cdot \bar{Z}_{q}(\Omega)}{\bar{Z}_{q}(\Omega)+\bar{Z}_{q}(\Omega)}+j \frac{\Omega \cdot L_{L}}{\sqrt{L_{1} \cdot C_{1}}}+R_{L}$.

Fig. 9 shows pullability exhibited by a single- and dual-crystal unit operated in series with load inductance. The inductance values were measured by the HP 4194A impedance/gain-phase analyzer. At the frequency of $19 \mathrm{MHz}$, the oscillator circuit has not been stable anymore.

The results show that when the crystal unit is inductively pulled, the frequency range could be made wider with larger inductance value, but the frequency stability gets worse rapidly with increasing inductance. As the frequency is varied, a sudden skip of the frequency with hysteresis may be observed. This phenomenon can be cured by putting a 10-30 killohm resistor in parallel to the inductor. Frequency stability also depends on the temperature coefficient of the core material used. The proper choice of the core material is also the key in the sense of the frequency stability. Table 2 shows a comparison of the oscillator's frequency stability for the capacitively- or inductively-pulled single- or double-crystal units. After 20 minutes, the oscillator exhibited a temperature drift of 0.01 Hz. 

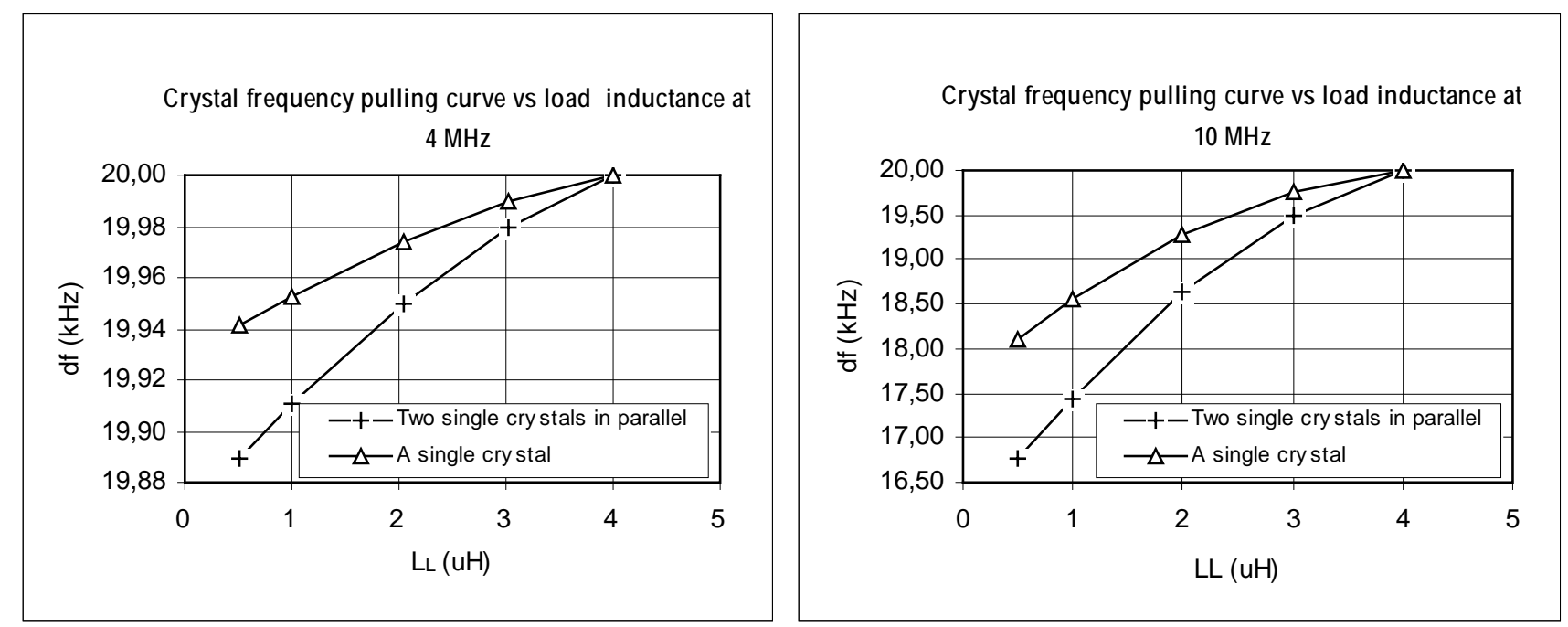

Figure 9. A comparison of pullability exhibited by a single- and dual-crystal unit operated in series with load inductance at the frequencies of 4 and $10 \mathrm{MHz}$ respectively.

Table 2. Frequency stability.

\begin{tabular}{|l|l|l|l|l|l|l|l|}
\hline Load & \multicolumn{3}{|c|}{ C } & \multicolumn{3}{c|}{} \\
\hline$f_{L}[\mathrm{MHz}]$ & 4 & 10 & 19 & 4 & 10 & 19 \\
\hline A single crystal & $\pm 0.01 \mathrm{~Hz}$ & $\pm 0.1 \mathrm{~Hz}$ & $\pm 0.2 \mathrm{~Hz}$ & $\pm 0.01 \mathrm{~Hz}$ & $\pm 0.1 \mathrm{~Hz}$ & - \\
\hline Two Single Crystals & $\pm 0.01 \mathrm{~Hz}$ & $\pm 0.1 \mathrm{~Hz}$ & $\pm 0.2 \mathrm{~Hz}$ & $\pm 0.01 \mathrm{~Hz}$ & $\pm 0.1 \mathrm{~Hz}$ & - \\
\hline
\end{tabular}

In general, the oscillator's circuit long-term stability also depends upon the crystal aging ( \pm 5 $\mathrm{ppm} /$ year), temperature stability $\left( \pm 3 \mathrm{ppm} /\left(-10^{\circ} \mathrm{C}\right.\right.$ to $\left.\left.+40{ }^{\circ} \mathrm{C}\right)\right)$ and the stability of the electronic circuit which depends upon the circuit type and quality of its elements. Another very important criterion for oscillator application is the drive level (power dissipation), which may not exceed $500 \mu \mathrm{W}$. Values higher than $500 \mu \mathrm{W}$ reduce the pulling range of the crystal. The maximum attainable stability of a crystal unit is also dependent on the $Q$ value [9-10].

\section{Conclusions}

Experimental results of the comparison of oscillators using a single-quartz crystal and those using two single-quartz crystals show that the use of two crystals of the same frequency increases the pulling range by 30-60\%. Depending on the circuit used, the pulling range can be increased up to 200\%. An extended pulling range of the crystal is achieved by changing capacitance or inductance external to the crystal unit. When the load capacitor is connected in series with the crystal, the frequency of operation of the oscillator is increased. In such case, the change in frequency is greater at lower values of load capacitance than at higher ones. Conversely, when an inductor is connected in series with the crystal the frequency operation is decreased. In both cases, the pulling function is nonlinear (Fig. 9). 
It should also be emphasized that the exact pulling limits depend on the crystal's $Q$-value as well as associated stray capacitances. The most common factors affecting frequency stability such as operating temperature range, aging and drive level as well as all other crystal characteristics influencing the stability should also be considered. Increased pulling range obtained experimentally can be used for determination of porosity using a water picnometer with capacitive level detection glass-fiber resins, measurements of small volumes and many other non-electrical quantities [11-13].

\section{References}

1. Schrüfer, E. Electrical Measurement: Quartz as a Frequency reference. Carl Hanser Verlag: München, Wien, 1992, p. 405 - 412.

2. Omig, SA. Quartz Crystal Theory: http://www.omig.pl/en/crystal_units.html.

3. Meeker, T. R. Theory and Properties of Piezoelectric Resonators and Waves: In Precision Frequency Control. Academic Press, 1985, vol. 1, p. 47 - 119.

4. Brice, J. C. Crystals for Quartz Resonators. Reviews of Modern Physics 1985, 57(1), 105-138.

5. Parzen, B. Design of Crystal and Other Harmonic Oscillators. Chapter 3: Piezoelectric Resonators. Wiley: New York, 1983.

6. Warner, A. W. Ultra - Precise Quartz Crystal Frequency Standards. IRE Transaction on Instrumentation 1958, 1-7, 185-188.

7. Miller, G. L.; Wagner, E. R. Resonant phase shift technique for the measurement of small changes in grounded capacitors. Review of Scientific Instruments 1990, 61 (4), 1267-1272.

8. Matko, V. Quartz sensor for water absorption measurement in glass - fiber resins. IEEE Transactions on Instrumentation and Measurement 1998, 47 (5), 1159-1162.

9. Kusters, J. A.; Vig, J. R. Thermal Hysteresis in Quartz Resonators. Proc. 44th Ann. Symposium Frequency Control, 1990. IEEE Catalog: No. 90CH2818-3, p. 165 - 175.

10. Rutman, J.; Walls, F. L. Characterization of Frequency Stability in Precision Frequency Sources. IEEE Transactions on Instrumentation and Measurement 1991, 79 (6), 952-960.

11. Matko, V. Determination of porosity using a water pycnometer with capacitive level detection. Sensors and Materials 2004, 16, 1-12.

12. Matko V.; Koprivnikar J. Measurement of 0 - $1 \mathrm{ml}$ volumes using the procedure of capacitive dependent crystals. Sensors and Actuators A 1993, 39, 111-116.

13. Tichy, J.; Gautschi, G. Piezomeasurement. Springer Verlag: Berlin - Heidelberg - New York, 1980.

(C) 2006 by MDPI (http://www.mdpi.org). Reproduction is permitted for noncommercial purposes. 